\title{
Length and area biased exponentiated weibull distribution based on forest inventories
}

\begin{abstract}
Length-biased and Area-biased distributions arise in many forestry applications, as well as other environmental, econometric, and biomedical sampling problems. We examine the Length-biased and Area-biased distributions versions of the Exponentiated Weibull distribution (EW). This study introduced a new distribution based on Length-biased Exponentiated Weibull distribution (LBEW) and Area-biased Exponentiated Weibull distribution (ABEW). Some characteristics of the new distributions were obtained. Plots for the cumulative distribution function, pdf and tables with values of skewness and kurtosis were also provided. Height-Diameter (H-D) data on Bombax and Pines (Pinus caribaea) were used to demonstrate the application of the distributions. Estimation of parameters of EW, LBEW and ABEW distributions were done using the maximum likelihood approach and compared across the distributions using criteria like AIC and Loglikelihood. We therefore proposed that similar to Exponentiated Weibull distribution (EW), a better fitting of Bombax and Pines H-D data are possible by LBEW and ABEW distributions. We hope in numerous fields of theoretical and applied sciences, the findings of this study will be useful for the practitioners.
\end{abstract}

Keywords: length-biased distribution, area-biased distributions, forestry, bombax, pines, height and diameter
Volume 6 Issue 2 - 2017

\author{
Oyamakin Samuel Oluwafemi, Durojaiye \\ Monsuru Olalekan \\ Department of Statistics, Faculty of Science, University of Ibadan, \\ Nigeria
}

Correspondence: Oyamakin Samuel Oluwafemi, Department of Statistics, Faculty of Science, University of Ibadan, Nigeria, Tel +2348066266535, Email fm_oyamakin@yahoo.com

Received: April 26, 2017 | Published: July II, 2017

\section{Introduction}

Trees contribute to the environment by providing oxygen, improving air quality, climate amelioration, conserving water, preserving soil, and supporting wildlife. During the process of photosynthesis, trees take in carbon dioxide and produce the oxygen we breathe. According to the U.S. Department of Agriculture, "One acre of forest absorbs six tons of carbon dioxide and puts out four tons of oxygen. This is enough to meet the annual needs of 18 people." Trees, shrubs and turf also filter air by removing dust and absorbing other pollutants like carbon monoxide, sulphur dioxide and nitrogen dioxide. After trees intercept unhealthy particles, rain washes them to the ground. Trees can add value to your home, help cool your home and neighborhood break the cold winds to lower your heating costs, and provide food for wildlife.

Height-diameter relationships are used to estimate the heights of trees measured for their diameter at breast height (DBH). Such relationship describes the correlation between height and diameter of the trees in a stand on a given date and can be represented by a linear or non-linear statistical model. In forest inventory designs diameter at breast height is measured for all trees within sample plots, while height is measured for only some selected trees, normally the dominant ones in terms of their DBH. In this study, the two species of trees considered explained thus;

i. Pinus caribaea: 'Pinus' is from the Greek word 'pinos' (pine tree), possibly from the Celtic term 'pin' or 'pyn' (mountain or rock), referring to the habitat of the pine. Pinus caribaea is a fine tree to $20-30 \mathrm{~m}$ tall, often $35 \mathrm{~m}$, with a diameter of $50-80 \mathrm{~cm}$ and occasionally up to $1 \mathrm{~m}$; trunk generally straight and well formed; lower branches large, horizontal and drooping; upper branches often ascending to form an open, rounded to pyramidal crown; young trees with a dense, pyramidal crown. Pinus caribaea is rated as moderately fire resistant. It tolerates salt winds and hence may be planted near the coast.

ii. Bombax costatum: 'Bombax' is derived from the Greek 'bombux', meaning silk, alluding to the dense wool-like floss covering the inner walls of the fruits and the seeds. Bombax costatum is a fire resisting tree of the savannas and dry woodlands from Senegal to central Africa, from Guinea across Ghana and Nigeria to southern Chad. Its tuberous roots act as water and/ or sugar storage facilities during long drought periods. Usually associated with Pterocarpus erinaceus, Daniellia oliveri, Cordyla pinnata, Parkia biglobosa, Terminalia macroptera and Prosopis africana.

\section{Length-biased and area-biased distribution}

When the probability of selecting an individual in a population is proportional to its magnitude, it is called length biased sampling. However, when observations are selected with probability proportional to their length, the resulting distribution is called length-biased. When dealing with the problem of sampling and selection from a lengthbiased distribution, the possible bias due to the nature of data-collection process can be utilized to connect the population parameters to that of the sampling distribution. That is, biased sampling is not always detrimental to the process of inference on population parameters. Inference based on a biased sample of a certain size may yield more information than that given by an unbiased sample of the same size, 
provided that the choice mechanism behind the biased sample is known. Statistical analysis based on length-biased samples has been studied in detail since the early 70 's. Size-biased distributions have been found to be useful in probability sampling designs for forestry and other related studies. These designs are classified into lengthbiased methods where sampling is done with probability proportional to some lineal measure and area-biased methods where units are selected into the sample with probability proportional to some real attributes. Hence, area-biased distribution is the square of the random variable of $\mathrm{X}$ or the second order power of size-biased distribution

The concept of length-biased was introduced by Cox in $1962 .{ }^{1}$ This concept is found in various applications in biomedical area such as family history and disease, survival analysis, intermediate events and latency period of AIDS due to blood transfusion. Many works were done to characterize relationships between original distributions and their length-biased versions. Patill and Rao expressed some basic distributions and their length-biased forms such as log-normal, gamma, pareto, beta distributions. Recently, many researches are applied to length-biased for lifetime distribution, lengthbiased weighted Weibull distribution, and length-biased weighted generalized Rayleigh distribution, length-biased beta distribution, and Bayes estimation of length- biased Weibull distribution. ${ }^{2}$

\section{Exponentiated weibull distribution}

The Weibull distribution was introduced by Wallodi Weibull, Swedish scientist, in 1951. It is perhaps the most widely used distribution to analyze the lifetime data. Gupta \& $\mathrm{Kundu}^{3}$ proposed an Exponentiated Exponential distribution which is a special case of the Exponentiated Weibull family. Flaih et al., ${ }^{4}$ extended the Inverted Weibull distribution to the Exponentiated Inverted Weibull (EIW) distribution by adding another shape parameter. This study suggested that the EIW distribution can provide a better fit to the real dataset than the IW distribution. Shittu, O I. and Adepoju, K A. ${ }^{5}$ the exponentiated Weibull was used as an alternative distribution that adequately describe the wind speed and thereby provide better representation of the potentials of wind energy.

Structural properties of exponentiated weibull distribution: According to Mudhokar, et al., ${ }^{6}$ the Exponentiated Weibull density function is defined as;

$$
f_{E W}(x ; k, \lambda, \alpha)=\frac{\alpha k x^{k-1}}{\lambda^{k}} \exp \left(-(x / \lambda)^{k}\right)\left(1-\exp \left(-(x / \lambda)^{k}\right)\right)^{\alpha-1}
$$

and the cdf is;

$$
F_{E W}(x ; k, \lambda, \alpha)=\left(1-\exp \left(-(x / \lambda)^{k}\right)\right)^{\alpha}
$$

$\alpha$ and $k$ are shape parameters; $\lambda$ is a scale parameter.

the $r^{\text {th }}$ moment of the exponentiated weibull is given as;

$$
E\left(x^{r}\right)=\alpha \lambda^{r} \Gamma\left(\frac{r}{k}+1\right) \sum_{j=0}^{\infty} \frac{(-1)^{j}\left(\begin{array}{l}
\alpha-1 \\
j
\end{array}\right)}{(1+j)^{\frac{r}{k}+1}}
$$
EW is

Where $\Gamma\left(\frac{r}{k}+1\right)=\int_{0}^{\infty} x \frac{r}{k} \exp (-x) \partial x$ at $\mathrm{r}=1$, the first moment of

$$
\mathrm{E}(\mathrm{x})=\alpha \lambda \Gamma\left(\frac{1}{k}+1\right) \sum_{j=0}^{\infty} \frac{(-1)^{j}\left(\begin{array}{l}
\alpha-1 \\
j
\end{array}\right)}{(1+j)_{k}^{\frac{1}{k+1}}}
$$

hus;

at $r=2$ is the second moment and the variance of $\mathrm{EW}$ is given

$$
\operatorname{Var}(x)=E\left(x^{2}\right)-[E(x)]^{2}
$$

$$
\operatorname{Var}(\mathrm{x})=\alpha \lambda^{2} \Gamma\left(\frac{2}{k}+1\right) \sum_{j=0}^{\infty} \frac{(-1)^{j}\left(\begin{array}{l}
\alpha-1 \\
j
\end{array}\right)}{(1+j)_{k}^{-+1}}-\alpha^{2} \lambda^{2} \Gamma^{2}\left(\frac{1}{k}+1\right)\left[\sum_{j=0}^{\infty} \frac{(-1)^{j}\left(\begin{array}{l}
\alpha-1 \\
j
\end{array}\right)}{(1+j)_{k}^{-+1}}\right]^{2}
$$

The skewness and kurtosis of EW

$$
\mathrm{k}_{3}=\frac{\alpha \lambda \Gamma\left(\frac{3}{k}+1\right) \sum_{j=0}^{\infty} \frac{(-1)^{j}\left(\begin{array}{l}
\alpha-1 \\
j
\end{array}\right)}{(1+j)_{k}^{\frac{3}{+}+1}}-\left[3 \alpha \lambda \Gamma\left(\frac{2}{k}+1\right) \sum_{j=0}^{\infty} \frac{(-1)^{j}\left(\begin{array}{l}
\alpha-1 \\
j
\end{array}\right)}{(1+j)_{k}^{\frac{2}{+}+1}}\right]\left[\alpha \lambda \Gamma\left(\frac{1}{k}+1\right) \sum_{j=0}^{\infty} \frac{(-1)^{j}\left(\begin{array}{l}
\alpha-1 \\
j
\end{array}\right)}{(1+j)_{k}^{\frac{1}{-+1}}}\right]+2 \alpha \lambda \Gamma\left(\frac{1}{k}+1\right) \sum_{j=0}^{\infty} \frac{(-1)^{j}\left(\begin{array}{l}
\alpha-1 \\
j
\end{array}\right)}{(1+j)_{k}^{\frac{1}{+}+1}}}{[\operatorname{Var}(\mathrm{x})]^{3 / 2}}
$$

\section{Materials and methods}

In this study, we propose two new distributions which are LBEW and ABEW distributions. We first provide a general definition of the Length-biased and Area-biased distributions which we subsequently reveal their pdfs.

Let $f(x ; \theta)$ be the pdf of the random variable $X$ and $\theta$ be the unknown parameter. The weighted distribution is defined as;

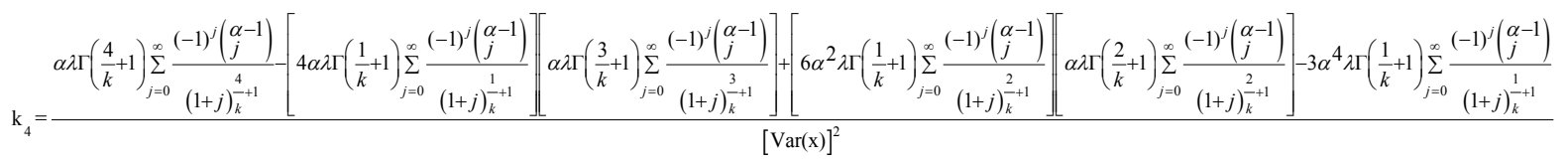




$$
g(x ; \theta)=\frac{x^{m} f(x ; \theta)}{E[f(x)]} \quad \begin{aligned}
& X \in R, \\
& \theta>0
\end{aligned}
$$

The distributions in equation (2) are termed as size-biased distribution of order $m$. When $m=1$, it is called size-biased of order 1 or say length biased distribution, whereas for $m=2$, it is called the area- biased distribution.

\section{Length-biased EW distribution (LBEW)}

If $X$ has a lifetime distribution with pdf $f(x)$ and expected value, $E[f(x)]<\infty$, the pdf of length-biased distribution of $X$ can be defined as:

$$
g_{L B}(x ; \theta)=\frac{x f(x ; \theta)}{E[f(x)]}
$$

Let $\mathrm{X}$ be a random variable of an EW distribution with pdf $f(x)$.

Then $g_{L B}(x ; \theta)=\frac{x f(x ; \theta)}{E[f(x)]}$ is a pdf of the LBEW distribution with two shape parameters $\alpha$ and k and a scale parameter $\lambda$. The notation for $X$ with the LBEW distribution is denoted as $\mathrm{X} \sim \mathrm{LBEW}$ ( $\alpha, k, \lambda)$. The pdf of $X$ is given by:

$$
g_{\text {LBEW }}(x ; k, \lambda, \alpha)=\frac{\frac{k x^{k}}{\lambda^{k+1}} \exp \left(-(x / \lambda)^{k}\right)\left(1-\exp \left(-(x / \lambda)^{k}\right)\right)^{\alpha-1}}{\Gamma\left(\frac{1}{k}+1\right) \sum_{j=0}^{\infty} \frac{(-1)^{j}\left(\begin{array}{l}
\alpha-1 \\
j
\end{array}\right)}{(1+j)_{k}^{-+1}}}
$$

\section{Area-biased EW distribution (ABEW)}

If $\mathrm{X}$ has a lifetime distribution with pdf $f(x)$ and expected value, $E[f(X)]<\infty$, the pdf of length-biased distribution of $X$ can be defined as:

$$
g_{A B}(x ; \theta)=\frac{x^{2} f(x ; \theta)}{E[f(x)]}
$$

Let $\mathrm{X}$ be a random variable of an EW distribution with pdf $f(x)$. Then $g_{A B}(x ; \theta)=\frac{x^{2} f(x ; \theta)}{E[f(x)]}$ is a pdf of the ABEW distribution with two shape parameters $\alpha$ and $k$ and a scale parameter $\lambda$. The notation for $X$ with the ABEW distribution is denoted as $X \sim A B E W$ ( $\alpha, k$ $, \lambda)$. The pdf of $X$ is given by:

$$
g_{A B E W}(x ; k, \lambda, \alpha)=\frac{\frac{k x^{k+1}}{\lambda^{k+1}} \exp \left(-(x / \lambda)^{k}\right)\left(1-\exp \left(-(x / \lambda)^{k}\right)\right)^{\alpha-1}}{\Gamma\left(\frac{1}{k}+1\right) \sum_{j=0}^{\infty} \frac{(-1)^{j}\left(\begin{array}{l}
\alpha-1 \\
j
\end{array}\right)}{(1+j)_{k}^{-+1}}}
$$

\section{The properties}

The LBEW distribution properties are as follows;

$$
\int_{0}^{\infty} g_{L B E W}(x) \partial x=\int_{0}^{\infty} \frac{\frac{k x^{k}}{\lambda^{k+1}} \exp \left(-(x / \lambda)^{k}\right)\left(1-\exp \left(-(x / \lambda)^{k}\right)\right)^{\alpha-1}}{\Gamma\left(\frac{1}{k}+1\right) \sum_{j=0}^{\infty} \frac{(-1)^{j}\left(\begin{array}{l}
\alpha-1 \\
j
\end{array}\right)}{(1+j)_{k}^{-+1}}} \partial x
$$

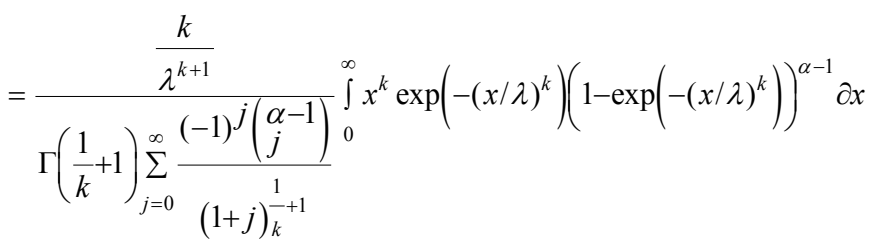

If $y=(x / \lambda)^{k}$, then we have that;

$$
=\frac{1}{\Gamma\left(\frac{1}{k}+1\right) \sum_{j=0}^{\infty} \frac{(-1)^{j}\left(\begin{array}{l}
\alpha-1 \\
j
\end{array}\right)}{(1+j)_{k}^{-+1}} \int_{0}^{\infty} y_{k}^{\frac{1}{k+2}} \exp (-y)(1-\exp (-y))^{\alpha-1} \partial y}
$$

Recall that,

$$
(1-\exp (-y))^{\alpha-1}=\sum_{j=0}^{\infty}(-1)^{j}\left(\begin{array}{l}
\alpha-1 \\
j
\end{array}\right) \exp (-y j)
$$

Therefore,

$$
\int_{0}^{\infty} g_{\text {LBEW }}(x) \partial x=\frac{1}{\Gamma\left(\frac{1}{k}+1\right) \sum_{j=0}^{\infty} \frac{(-1)^{j}\left(\begin{array}{l}
\alpha-1 \\
j
\end{array}\right)}{(1+j)_{k}^{-+1}}} \int_{0}^{\infty} y_{k}^{\frac{1}{k}+2} \sum_{j=0}^{\infty}(-1)^{j}\left(\begin{array}{l}
\alpha-1 \\
j
\end{array}\right) \exp (-y[1+j]) \partial y
$$

; where $m=y[1+j]$

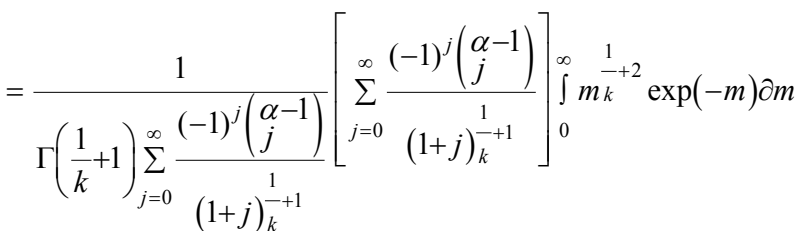

$\int_{0}^{\infty} g_{\text {LBEW }}(x) \partial x=\frac{1}{\Gamma\left(\frac{1}{k}+1\right) \sum_{j=0}^{\infty} \frac{(-1)^{j}\left(\begin{array}{l}\alpha-1 \\ j\end{array}\right)}{(1+j)_{k}^{\frac{1}{++1}}}}\left[\sum_{j=0}^{\infty} \frac{(-1)^{j}\left(\begin{array}{l}\alpha-1 \\ j\end{array}\right)}{(1+j)_{k}^{-\frac{1}{2}}}\right] \Gamma\left(\frac{1}{k}+1\right)$

$\int_{0}^{\infty} g_{\text {LBEW }}(x) \partial x=1$

Therefore, the pdf of LBEW distribution sum to 1. NB: It was also obtainable for the ABEW distribution.

The cdf of LBEW, corresponding to (4) is obtained by 


$$
\begin{aligned}
& F_{\text {LBEW }}(X)=\int_{0}^{x} \frac{\frac{k x^{k}}{\lambda^{k+1}} \exp \left(-(x / \lambda)^{k}\right)\left(1-\exp \left(-(x / \lambda)^{k}\right)\right)^{\alpha-1}}{\Gamma\left(\frac{1}{k}+1\right) \sum_{j=0}^{\infty} \frac{(-1)^{j}\left(\begin{array}{l}
\alpha-1 \\
j
\end{array}\right)}{(1+j)_{k}^{-+1}}} \partial x \\
& F_{L B E W}(X)=\frac{\frac{k}{\lambda^{k+1}}}{\Gamma\left(\frac{1}{k}+1 \sum_{j=0}^{\infty} \frac{(-1)^{j}\left(\begin{array}{l}
\alpha-1 \\
j
\end{array}\right)}{(1+j)_{k}^{-+1}} \int^{x} x^{k} \exp \left(-(x / \lambda)^{k}\right)\left(1-\exp \left(-(x / \lambda)^{k}\right)\right)^{\alpha-1} \partial x\right.}
\end{aligned}
$$

Let $y=(x / \lambda)^{k} ; y \lambda^{k}=x^{k} ; x=\lambda y^{1 / k}$

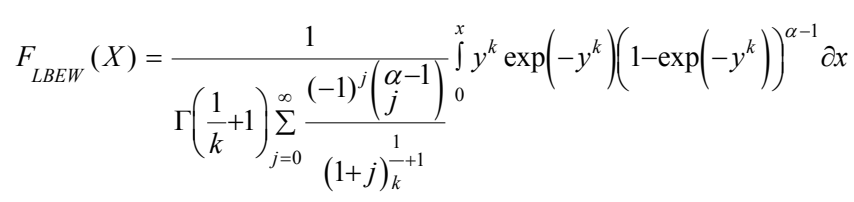

Let $m=y(1+j) ; y=\frac{m}{1+j}$

$$
\begin{aligned}
& F_{L B E W}(X)=\frac{1}{\Gamma\left(\frac{1}{k}+1\right) \int_{0}^{x} m^{1 / k} \exp (-m) \partial m} \\
& F_{L B E W}(X)=\frac{x^{1 / k} \exp (-x)\left[1+\frac{1}{k x}\right]}{\Gamma\left(\frac{1}{k}+1\right)\left[1-\frac{1}{k}+\frac{1}{k^{2}}\right]}
\end{aligned}
$$

So, the reliability function of $\mathrm{LBEW}$ is,

$$
\begin{aligned}
& R(x)=1-F_{L B E W}(x)=1-\frac{x^{1 / k} \exp (-x)\left[1+\frac{1}{k x}\right]}{\Gamma\left(\frac{1}{k}+1\right)\left[1-\frac{1}{k}+\frac{1}{k^{2}}\right]} \\
& R(x)=\frac{\Gamma\left(\frac{1}{k}+1\right)\left[1-\frac{1}{k}+\frac{1}{k^{2}}\right]-\left[1+\frac{1}{k x}\right] x^{1 / k} \exp (-x)}{\Gamma\left(\frac{1}{k}+1\right)\left[1-\frac{1}{k}+\frac{1}{k^{2}}\right]}
\end{aligned}
$$

And the hazard function is,

$$
h(x)=\frac{f(x)}{R(x)}=\frac{\frac{k x^{k}}{\lambda^{k+1}} \exp \left(-(x / \lambda)^{k}\right)\left(1-\exp \left(-(x / \lambda)^{k}\right)\right)^{\alpha-1}\left[1-\frac{1}{k}+\frac{1}{k^{2}}\right]}{\left\{\Gamma\left(\frac{1}{k}+1\right)\left[1-\frac{1}{k}+\frac{1}{k^{2}}\right]-\left[1+\frac{1}{k x}\right] x^{1 / k} \exp (-x)\right\} \sum_{j=0}^{\infty} \frac{(-1)^{j}\left(\begin{array}{l}
\alpha-1 \\
j
\end{array}\right)}{(1+j)_{k}^{-+1}}}
$$

\section{The moments}

The $\boldsymbol{r}$ th raw moment of the LBEW random variable $\mathrm{X}$ is

$$
\begin{aligned}
& E\left(X^{r}\right)=\frac{1}{\Gamma\left(\frac{1}{k}+1\right) \sum_{j=0}^{\infty} \frac{(-1)^{j}\left(\begin{array}{c}
\alpha-1 \\
j
\end{array}\right)}{(1+j) \frac{1}{k}+1} \int_{0}^{\infty} x^{r} \frac{\alpha k x^{k}}{\lambda^{k+1}} \exp \left(-(x / \lambda)^{k}\right)\left(1-\exp \left(-(x / \lambda)^{k}\right)\right)^{\alpha-1} \partial x} \\
& E\left(X^{r}\right)=\frac{1}{\lambda^{r} \Gamma\left(\frac{1}{k}+1\right)} \Gamma\left(\frac{r+1}{k}+1\right) \sum_{j=0}^{\infty} \frac{(1+j)^{(1 / k)+1}}{(1+j)^{((r+1) / k)+1}}
\end{aligned}
$$

at $r=1$, the first moment of LBEW is

$$
E(X)=\frac{1}{\lambda \Gamma\left(\frac{1}{k}+1\right)} \Gamma\left(\frac{2}{k}+1\right) \sum_{j=0}^{\infty}(1+j)^{-(1 / k)}
$$

And the variance is

$$
\operatorname{Var}(X)=\frac{1}{\lambda^{2} \Gamma\left(\frac{1}{k}+1\right)} \Gamma\left(\frac{3}{k}+1\right) \sum_{j=0}^{\infty}(1+j)^{-(1 / k)}-\left[\frac{1}{\lambda \Gamma\left(\frac{1}{k}+1\right)} \Gamma\left(\frac{2}{k}+1\right) \sum_{j=0}^{\infty}(1+j)^{-(1 / k)}\right]^{2}
$$

The skewness and kurtosis of LBEW;

$$
\begin{aligned}
& k_{3}=\frac{\alpha \lambda \Gamma\left(\frac{3}{k}+1\right) \sum_{j=0}^{\infty} \frac{(-1)^{j}\left(\begin{array}{l}
\alpha-1 \\
j
\end{array}\right)}{(1+j)}-\frac{3}{k}+1}{k}\left[6 \alpha^{2} \lambda \Gamma\left(\frac{4}{k}+1\right) \sum_{j=0}^{\infty} \frac{(-1)^{j}\left(\begin{array}{l}
\alpha-1 \\
j
\end{array}\right)}{(1+j) \frac{4}{k}+1}\right]\left[\alpha^{2} \lambda \Gamma\left(\frac{2}{k}+1\right) \sum_{j=0}^{\infty} \frac{(-1)^{j}\left(\begin{array}{l}
\alpha-1 \\
j
\end{array}\right)}{(1+j) \frac{2}{k}+1}\right]+8 \alpha^{2} \lambda^{2} \Gamma\left(\frac{1}{k}+1\right) \sum_{j=0}^{\infty} \frac{(-1)^{j}\left(\begin{array}{l}
\alpha-1 \\
j
\end{array}\right)}{(1+j) \frac{1}{k}+1} \\
& \left.k_{4}=\frac{\alpha \lambda^{2} \Gamma\left(\frac{4}{k}+1\right) \sum_{j=0}^{\infty} \frac{(-1)^{j}\left(\begin{array}{l}
\alpha-1 \\
j
\end{array}\right)}{(1+j) \frac{4}{k}+1}-\left[8 \alpha^{2} \lambda^{2} \Gamma\left(\frac{3}{k}+1\right) \sum_{j=0}^{\infty} \frac{(-1)^{j}\left(\begin{array}{l}
\alpha-1 \\
j
\end{array}\right)}{(1+j) \frac{3}{k}+1}\right]\left[\alpha^{2} \lambda \Gamma\left(\frac{4}{k}+1\right) \sum_{j=0}^{\infty} \frac{(-1)^{j}\left(\begin{array}{l}
\alpha-1 \\
j
\end{array}\right)}{(1+j) \frac{4}{k}+1}\right]+\left[6 \alpha^{2} \lambda^{2} \Gamma\left(\frac{2}{k}+1\right) \sum_{j=0}^{\infty} \frac{(-1)^{j}}{\left(\begin{array}{l}
\alpha \\
\left(\begin{array}{l}
\alpha \\
j
\end{array}\right.
\end{array}\right)}\right.}{(1+j) \frac{2}{k}+1}\right]
\end{aligned}
$$

From equation (7), (8), (9), (10), (11), (12), (13), (14), we established all the properties of LBEW distribution and also that of ABEW was obtained which can be fetch in the body of the work. 


\section{Maximum likelihood approach}

Harter and Moore (1965) were the earliest statisticians to use the maximum likelihood procedure because of its desirable characteristics.

The three distributions in the study (EW, LBEW and ABEW) are solved iteratively by computer algorithm to obtain the maximum likelihood estimates of the parameters $\alpha, \mathrm{k}$ and $\lambda$.

\section{MLE of EW}

Let $\mathrm{X}$ be a random sample of size $n$ from the EW distribution given by equation (1). Then the log likelihood function comes out to be

$$
\begin{aligned}
& L(\alpha, \lambda, k)=n \ln \alpha+n \ln k+n k \ln \lambda+(k-1) \sum \ln x_{i}+ \\
& \quad(\alpha-1) \sum \ln \left[1-\exp \left\{-\left(x_{i} / \lambda\right)^{k}\right\}\right]-\sum\left(x_{i} / \lambda\right)^{k}
\end{aligned}
$$

Therefore the MLEs of $\alpha, \lambda, \mathrm{k}$ which maximize (15) must satisfy the normal equations given by

Derivative w.r.t $\alpha$

$$
\frac{\partial}{\partial \alpha} L(\alpha, \lambda, k)=\frac{n}{\alpha}+\sum \ln \left[1-\exp \left\{-\left(x_{i} / \lambda\right)^{k}\right\}\right]=0
$$

We obtain the MLE of $\alpha$ as

$$
\alpha=-\frac{n}{\sum \ln \left[1-\exp \left\{-\left(x_{i} / \lambda\right)^{k}\right\}\right]}
$$

Derivative w.r.t $\lambda$

$$
\frac{\partial}{\partial \lambda} L(\alpha, \lambda, k)=\frac{n k}{\lambda}+(\alpha-1) k \lambda^{k-1} \sum \frac{\exp \left\{-\left(x_{i} / \lambda\right)^{k}\right\}}{1-\exp \left\{-\left(x_{i} / \lambda\right)^{k}\right\}} x_{i}-k \lambda^{k-1} \sum x_{i}^{k}=0
$$

Multiplying the above equation by $\frac{\lambda}{k}$ we get

$$
n+\lambda^{k}\left[(\alpha-1) \sum \frac{\exp \left\{-\left(x_{i} / \lambda\right)^{k}\right\}}{1-\exp \left\{-\left(x_{i} / \lambda\right)^{k}\right\}} x_{i}^{k}-\sum x_{i}^{k}\right]=0
$$

Derivative w.r.t k

$\frac{\partial}{\partial k} L(\alpha, \lambda, k)=\frac{n}{k}+n \ln \lambda+\sum \ln x_{i}+(\alpha-1) \lambda^{k} \sum \frac{\exp \left\{-\left(x_{i} / \lambda\right)^{k}\right\}}{1-\exp \left\{-\left(x_{i} / \lambda\right)^{k}\right\}} x^{k} \ln \left(x_{i} / \lambda\right)-\lambda^{k-1} \sum x_{i}{ }^{k} \ln \left(x_{i} / \lambda\right)=0$ then,

$$
\frac{n}{k}+\sum \ln x_{i}+\lambda^{k}\left[(\alpha-1) \sum \frac{\exp \left\{-\left(x_{i} / \lambda\right)^{k}\right\}}{1-\exp \left\{-\left(x_{i} / \lambda\right)^{k}\right\}} x_{i}^{k} \ln x_{i}-\sum x_{i}^{k} \ln x_{i}\right]=0
$$

Using (15) in (17) and (18) we get equations, which are satisfied by the MLEs $\hat{\lambda}$ and $\hat{k}$ of $\lambda$ and $k$, respectively. Because of the complicated form of the likelihood equations, algebraically it is very difficult to prove that the solution to the normal equations give a global maximum or at least a local maximum, though numerical computation during data analysis showed the presence of at least local maximum.

However, the following properties of the log-likelihood function have been algebraically noted:

for given $(\lambda, k)$, log-likelihood is a strictly concave function of $\alpha$. Further, the optimal value of $\alpha$, given by (8), is a concave increasing function of $\lambda$, for given $k$;

for given ( $\alpha, k)$, and $\alpha \geq 1, \log$-likelihood is a strictly concave function of $\lambda$.

\section{MLE of LBEW}

Taking the log-likelihood and derivative of the equation (4) to obtain the MLEs of parameters $\alpha, \mathrm{k}$ and $\lambda$

$$
\begin{aligned}
& \frac{\partial}{\partial \alpha} L(\alpha, \lambda, k)=\sum_{i=1}^{n}(\alpha-1) \ln \left[1-\exp \left\{-\left(x_{i} / \lambda\right)^{k}\right\}\right]^{\alpha-2}-\frac{\partial}{\partial \alpha}\left\{n \ln \left[\Gamma\left(\frac{1}{k}+1\right) \sum_{j=0}^{\infty} \frac{(-1)^{j}\left(\begin{array}{l}
\alpha-1 \\
j
\end{array}\right)}{(1+j) \frac{1}{k}+1}\right]\right\}=0 \\
& \frac{\partial}{\partial k} L(\alpha, \lambda, k)=\frac{n}{k}+\sum_{i=1}^{n} \frac{k \ln x_{i}^{k-1}}{x_{i}^{k}}-n \ln \lambda-k(x / \lambda)^{k}-\sum_{i=1}^{n}\left\{\frac{(\alpha-1) \exp \left\{-\left(k x_{i}^{k} / \lambda^{k+1}\right)\right\}}{1-\exp \left\{-\left(x_{i} / \lambda\right)^{k}\right\}}\right\}^{\alpha-1}-\frac{\partial}{\partial \alpha}\left\{n \ln \left[\Gamma\left(\frac{1}{k}+1\right) \sum_{j=0}^{\infty} \frac{(-1)^{j}\left(\begin{array}{l}
\alpha-1 \\
j
\end{array}\right)}{(1+j) \frac{1}{k}+1}\right]\right\}=0 \\
& \frac{\partial}{\partial \lambda} L(\alpha, \lambda, k)=-\frac{n(k+1)}{\lambda}+\frac{k x_{i}{ }^{k}}{\lambda^{k+1}}-\sum_{i=1}^{n}\left\{\frac{(\alpha-1) \exp \left\{-\left(k x_{i}{ }^{k} / \lambda^{k+1}\right)\right\}}{1-\exp \left\{-\left(x_{i} / \lambda\right)^{k}\right\}}\right\}^{\alpha-1}=0
\end{aligned}
$$

Equations (19), (20) and (21) are solved iteratively to obtain the maximum likelihood estimates of the parameters $\alpha, \mathrm{k}$ and $\lambda$.

\section{MLE of ABEW}

Taking the log-likelihood and derivative of the equation (6) to obtain the MLEs of parameters $\alpha, \mathrm{k}$ and $\lambda$

$$
\frac{\partial}{\partial \alpha} L(\alpha, \lambda, k)=\sum_{i=1}^{n}(\alpha-1) \ln \left[1-\exp \left\{-\left(x_{i} / \lambda\right)^{k}\right\}\right]^{\alpha-2}-\frac{\partial}{\partial \alpha}\left\{n \ln \left[\Gamma\left(\frac{1}{k}+1\right) \sum_{j=0}^{\infty} \frac{(-1)^{j}\left(\begin{array}{l}
\alpha-1 \\
j
\end{array}\right)}{(1+j) \frac{1}{k}+1}\right]\right\}=0
$$




$$
\begin{gathered}
\frac{\partial}{\partial k} L(\alpha, \lambda, k)=\frac{n}{k}+\sum_{i=1}^{n} \frac{(k+1) \ln x_{i}^{k}}{x_{i}^{k+1}}-n \ln \lambda-k(x / \lambda)^{k}-\sum_{i=1}^{n}\left\{\frac{(\alpha-1) \exp \left\{-\left(k x_{i}^{k} / \lambda^{k+1}\right)\right\}}{1-\exp \left\{-\left(x_{i} / \lambda\right)^{k}\right\}}\right\}^{\alpha-1}-\frac{\partial}{\partial \alpha}\left\{n \ln \left[\Gamma\left(\frac{1}{k}+1\right) \sum_{j=0}^{\infty} \frac{\left.(-1)^{j}\left(\begin{array}{l}
\alpha-1 \\
j
\end{array}\right)\right]}{(1+j) \frac{1}{k}+1}\right]\right\}=0 \\
\frac{\partial}{\partial \lambda} L(\alpha, \lambda, k)=-\frac{(k+1)}{\lambda}+\frac{k x_{i}^{k}}{\lambda^{k+1}}-\sum_{i=1}^{n}\left\{\frac{(\alpha-1) \exp \left\{-\left(k x_{i}^{k} / \lambda^{k+1}\right)\right\}}{1-\exp \left\{-\left(x_{i} / \lambda\right)^{k}\right\}}\right\}^{\alpha-1}=0
\end{gathered}
$$

Also, equations (22), (23) and (24) are solved iteratively to obtain the maximum likelihood estimates of the parameters $\alpha, \mathrm{k}$ and $\lambda$.

\section{AIC and log-likelihood}

We calculate AIC value for each model with the same dataset, and the best model is the one with minimum AIC value. The value of AIC depends on the data Pines and Bombax, which leads to model selection uncertainty.

$$
A I C=-2 \log L\left(\hat{\theta} \mid x_{i}\right)+2 k
$$

where

$\stackrel{\Lambda}{\Lambda}\left(\hat{\theta} \mid x_{i}\right)=$ the maximized value of the likelihood function of the model, and where $\hat{\theta}$ are the parameter values that maximize the likelihood function;

$$
\begin{aligned}
x_{i} & =\text { the observed data; } \\
\mathrm{k} & =\text { the number of free parameters to be estimated. }
\end{aligned}
$$

\section{Results and discussion}

\section{Summary of the data}

The Bombax and Pine Height-Diameter data were extracted from the Forestry Research Institute of Nigeria's records, cleaned up and the summary statistics of the data was computed as presented in Tables 1 and 2.

\section{Maximum likelihood approach}

The above Table 3-5 shown the parameters estimation of EW, LBEW and ABEW distributions. We observed the comparison of the three distributions by their corresponding AIC and -2log-likelihood of each of the dataset pines and bombax. The ideal distribution is the one with the minimum AIC values (Figures 1-5)..$^{7-15}$

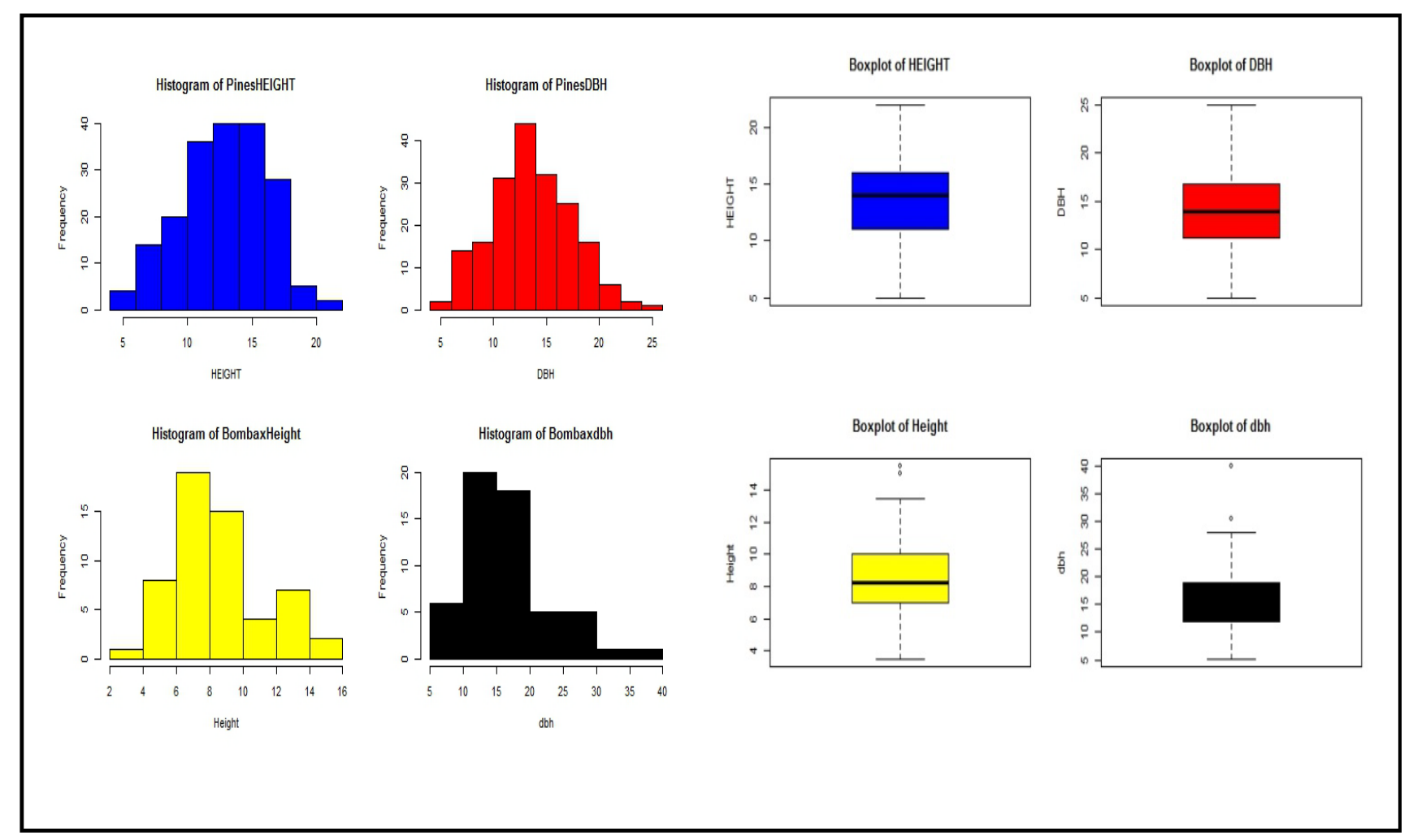

Figure I Histogram boxplot plot of bombax and pines H-D. 

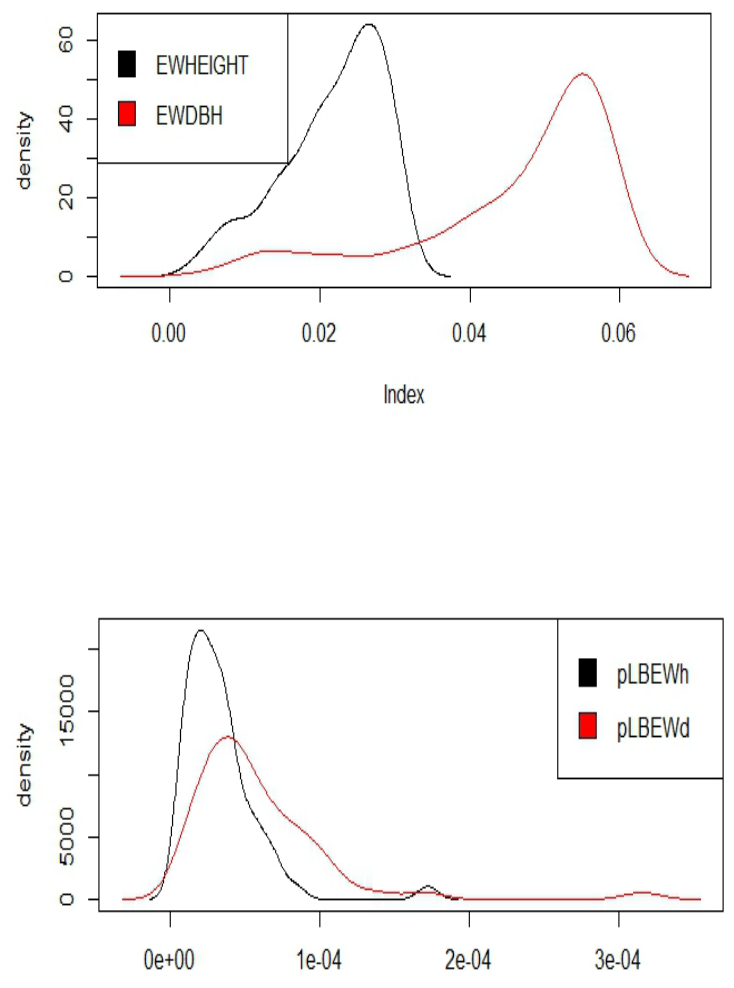

Index

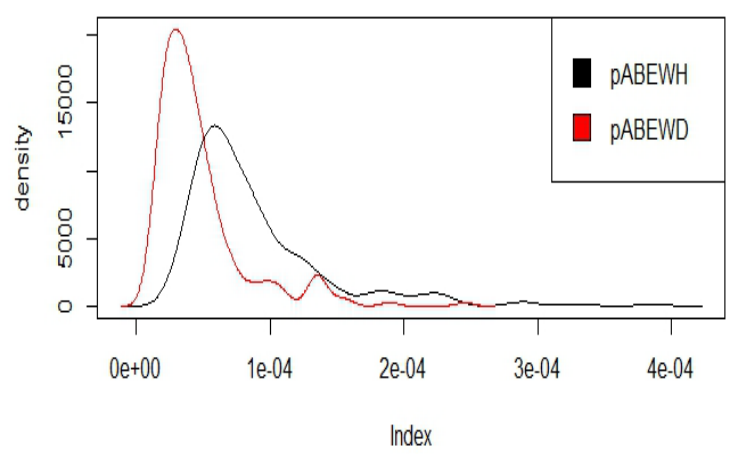

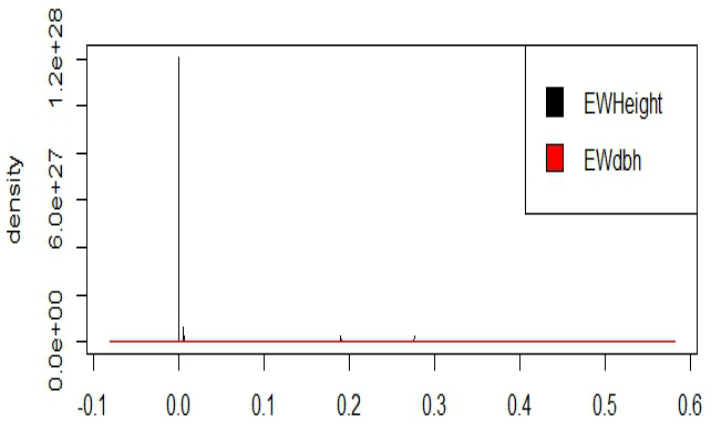

Index

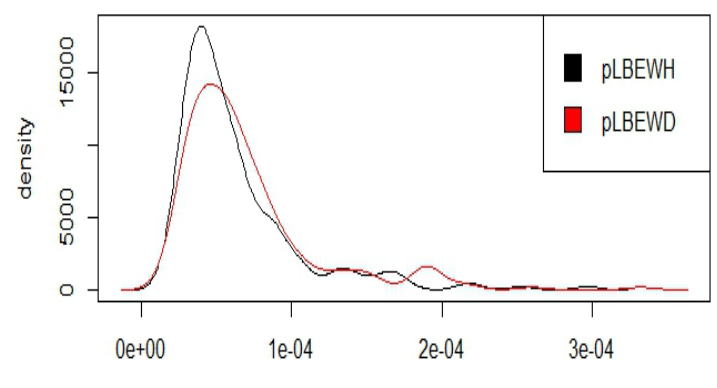

Index

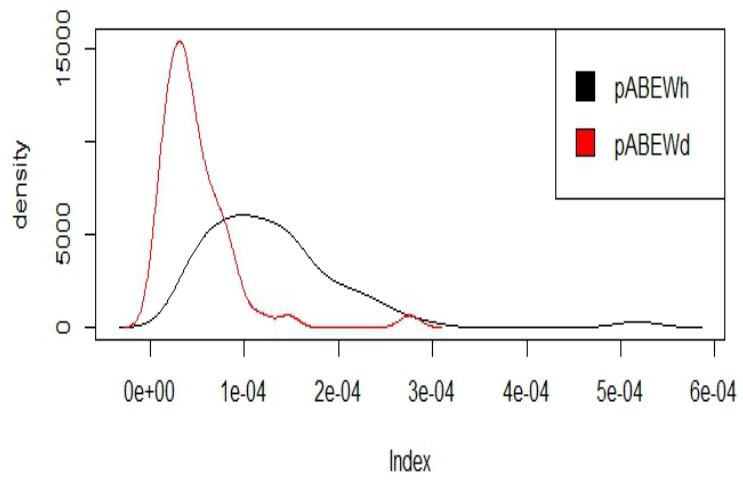

Figure $\mathbf{2}$ The probability distribution function of the EW, LBEW and ABEW distribution.

Table I Descriptive statistics of the data

\begin{tabular}{|c|c|c|c|c|}
\hline & PinesHEIGHT & PinesDBH & Bombaxheight & Bombaxdbh \\
\hline Mean & 13.33333 & I3.87566 & 8.82142 & 16.47857 \\
\hline estimated stdev & 3.38336 & 3.84751 & 2.67723 & 6.37982 \\
\hline estimated skewness & -0.14219 & 0.11252 & 0.6269 & 1.22949 \\
\hline estimated kurtosis & 2.683 & 2.76197 & 2.85557 & 5.31719 \\
\hline
\end{tabular}




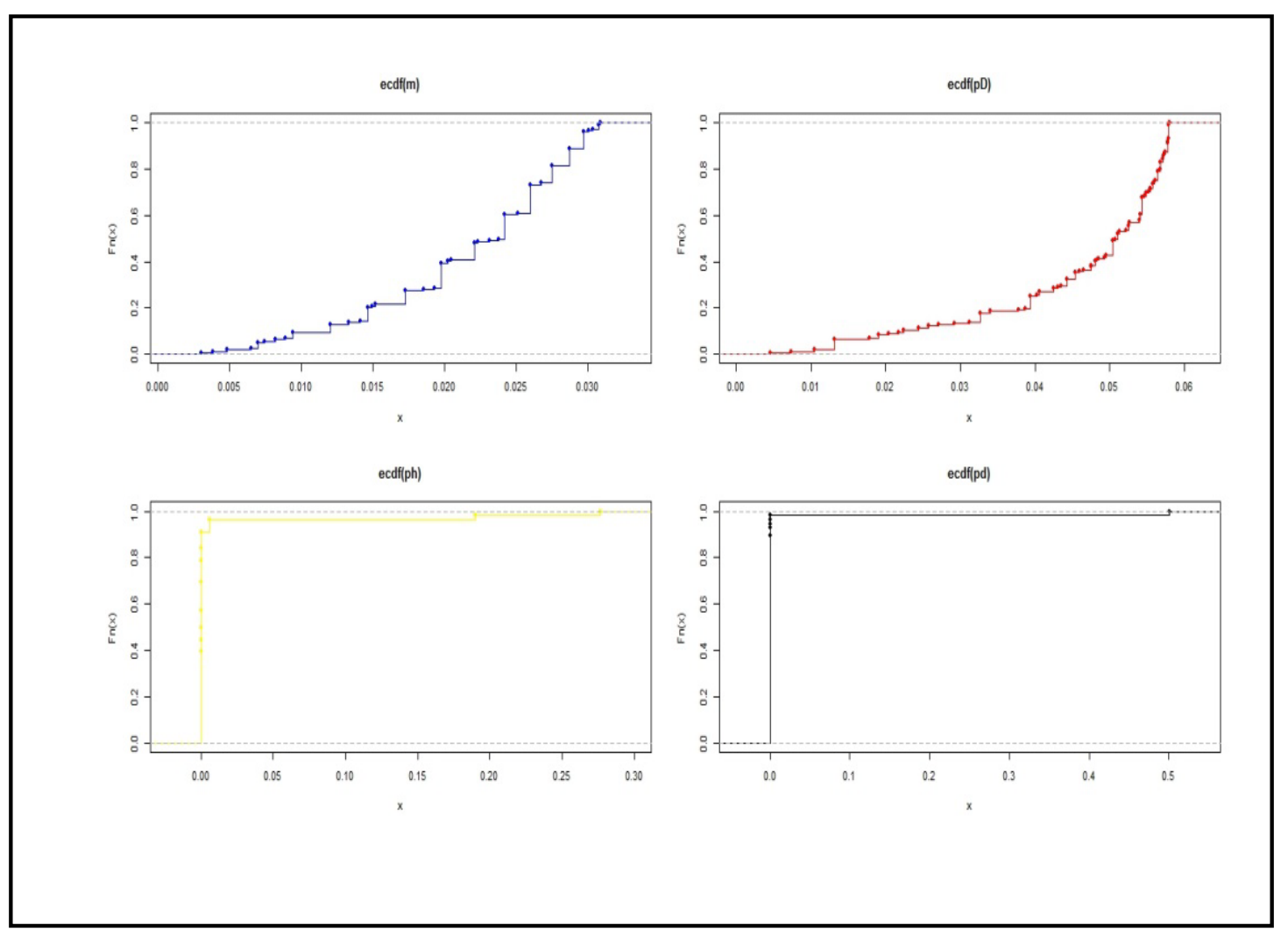

Figure 3 The cumulative distribution function of the EW distribution.

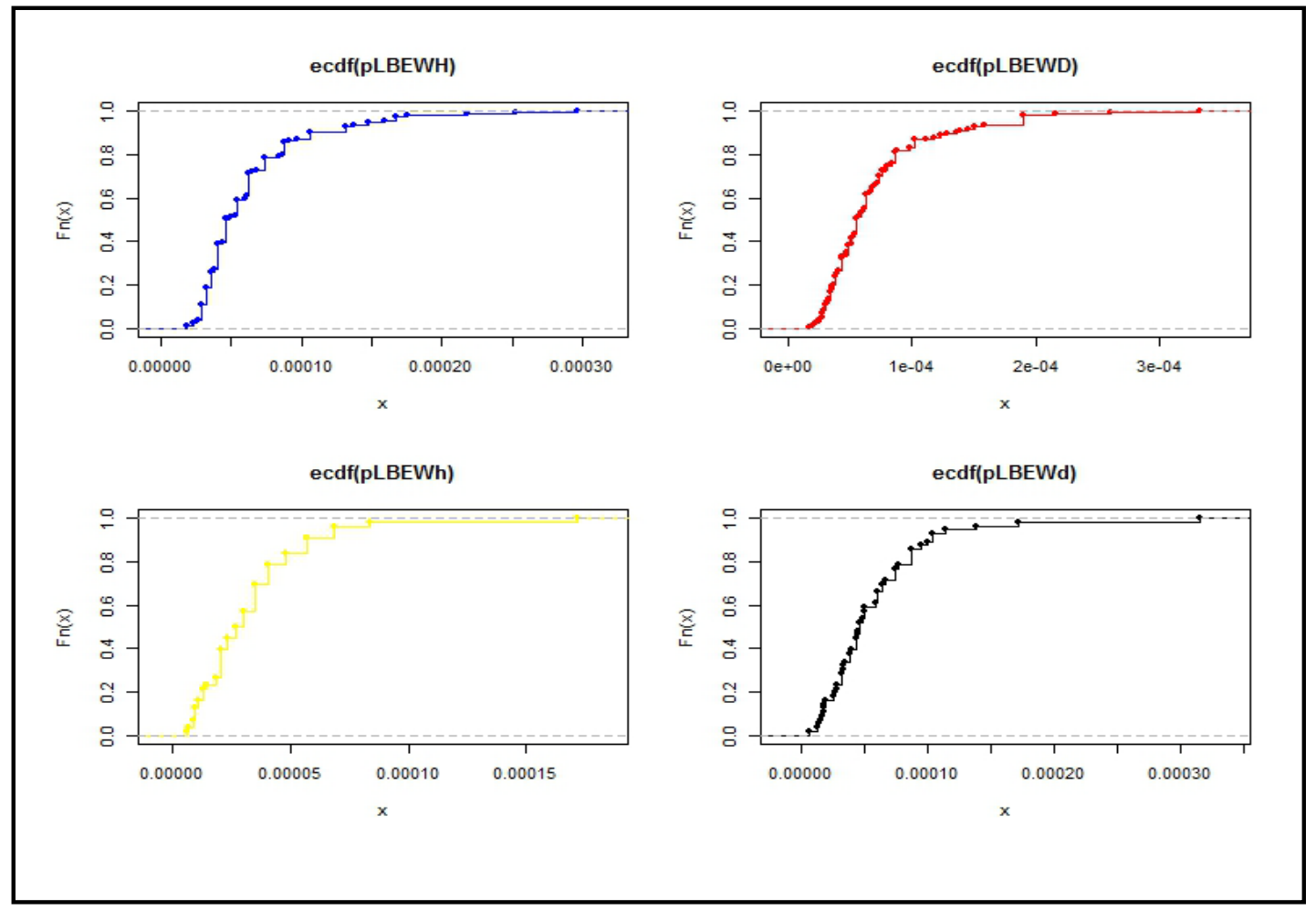

Figure 4 The cumulative distribution function of the LBEW distribution. 


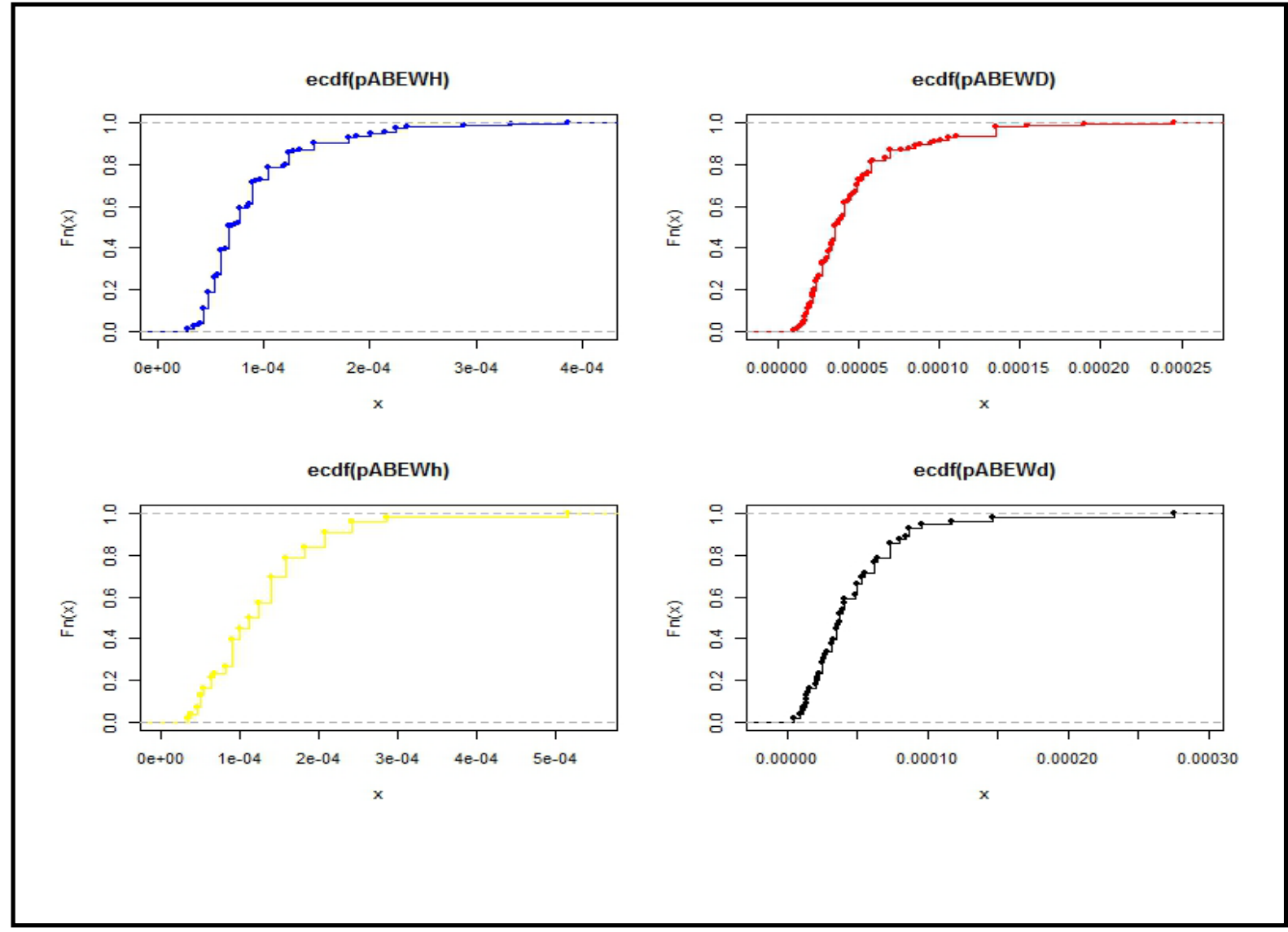

Figure $\mathbf{5}$ The cumulative distribution function of the ABEW distribution.

Table 2 Skewness and kurtosis of EW, LBEW and ABEW distribution

\begin{tabular}{|c|c|c|c|c|c|}
\hline EW & PinesHEIGHT & PinesDBH & Bombaxheight & Bombaxdbh & \\
\hline Mean & 0.02165 & 0.046100 & $0.00864 I$ & 0.008940 & 0.00685 \\
\hline estimated stdev & 0.013384 & 0.044389 & 0.066902 & & \\
\hline estimated skewness & -0.77246 & -1.379207 & 5.268808 & 7.281359 & \\
\hline estimated kurtosis & 2.757014 & 3.945957 & 29.65974 & 54.01818 & \\
\hline \multicolumn{6}{|l|}{ LBEW } \\
\hline Mean & $6.34 \mathrm{E}-05$ & 7.07E-05 & $3.32 \mathrm{E}-05$ & $5.88 \mathrm{E}-05$ & $4.28 \mathrm{E}-05$ \\
\hline estimated stdev & $4.78 \mathrm{E}-05$ & $2.62 \mathrm{E}-05$ & $4.82 \mathrm{E}-05$ & & \\
\hline estimated skewness & 2.408499 & 2.212744 & 2.871012 & 3.017609 & \\
\hline estimated kurtosis & 10.28893 & 9.160734 & 15.27437 & 15.87016 & \\
\hline \multicolumn{6}{|l|}{ ABEW } \\
\hline Mean & 8.97E-05 & $4.76 \mathrm{E}-05$ & 0.000130 & $4.88 \mathrm{E}-05$ & \\
\hline estimated stdev & $5.58 \mathrm{E}-05$ & 3.5IE-05 & $7.95 \mathrm{E}-05$ & 4.20E-05 & \\
\hline estimated skewness & 2.299099 & 2.336502 & 2.215247 & 3.142198 & \\
\hline estimated kurtosis & 9.618972 & 9.925224 & 11.11609 & $16.7262 \mid$ & \\
\hline
\end{tabular}


Table 3 Parameters estimation of EW

\begin{tabular}{|c|c|c|c|c|}
\hline \multirow{2}{*}{$\begin{array}{l}\text { EW } \\
\text { parameters }\end{array}$} & \multicolumn{2}{|l|}{ Pines } & \multicolumn{2}{|l|}{ Bombax } \\
\hline & HEIGHT & DBH & Height & dbh \\
\hline$k$ & 4.351 & 3.077 & 1.433 & 1.064 \\
\hline$\alpha$ & 1.053 & 1.635 & 8.920 & 11.725 \\
\hline$\lambda$ & 14.468 & 13.542 & 4.352 & 5.747 \\
\hline AIC & 1999.52 & 2098.4 & 538.40 & 719.45 \\
\hline$-2 \log l i k$ & 993.76 & 1043.2 & 263.20 & 353.72 \\
\hline \multicolumn{5}{|c|}{ Table 4 Parameters estimation of LBEW } \\
\hline LBEW & Pines & & Bombax & \\
\hline parameters & HEIGHT & DBH & Height & dbh \\
\hline$k$ & 0.05 & 0.05 & 0.05 & 0.05 \\
\hline$\alpha$ & 0.264 & 0.261 & 0.263 & 0.261 \\
\hline$\lambda$ & 0.045 & 0.052 & 0.01 & 0.053 \\
\hline AIC & 1629.07 & 1634.36 & 467.19 & 484.93 \\
\hline$-2 \log l i k$ & 407.117 & 408.44 & 116.65 & 121.08 \\
\hline \multicolumn{5}{|c|}{ Table 5 Parameters estimation of ABEW } \\
\hline ABEW & Pines & & Bomba & \\
\hline parameters & HEIGHT & DBH & Height & $\mathrm{dbh}$ \\
\hline$k$ & 0.050 & 0.050 & 0.050 & 0.050 \\
\hline$\alpha$ & 0.262 & 0.262 & 0.267 & 0.263 \\
\hline$\lambda$ & 0.068 & 0.034 & 0.045 & 0.045 \\
\hline AIC & 1533.11 & 1508.10 & 449.67 & 450.24 \\
\hline$-2 \log l i k$ & 383.128 & 376.874 & II 2.27 & II2.4I \\
\hline
\end{tabular}

\section{Conclusion}

This study introduced a new distribution based on LBEW and ABEW. Some characteristics of the new distributions were obtained. Plots for the cumulative distribution function, pdf and tables with values of skewness and kurtosis were also provided. HeightDiameter (H-D) data on Bombax and Pines (Pinus caribeae) were used to demonstrate the application of the distributions. Estimation of parameters of EW, LBEW and ABEW distributions were done using the maximum likelihood approach and compared across the distributions using criteria like AIC and Log-likelihood. We therefore proposed that similar to Exponentiated Weibull distribution (EW), a better fitting of Bombax and Pines H-D data are possible by LBEW and $\mathrm{ABEW}$ distributions.

\section{Acknowledgements}

We gratefully acknowledge the suggestions given by the anonymous referees, which have immensely helped to improve the presentation of the paper.

\section{Conflicts of interest}

None.

\section{References}

1. Cox D. Renewal Theory, Barnes and Noble, New York, USA. 1962.

2. Patil GP, Rao CR. Weighted distributions and size-biased sampling with applications to wildlife populations and human families. Biometrics. 1978;34(2):179-189.

3. Gupta R, Kundu D. Generalized exponential distributions. Australian and New Zealand Journal of Statistics. 1999;41(2):173-188.

4. Flaih A, Elsalloukh H, Mendi E, et al. The exponentiated inverted Weibull distribution. Applied Mathematics and Information Sciences. 2012;6(2):167-171.

5. Olanrewaju SI, Adepoju KA. On the Exponentiated Weibull Distribution for Modeling Wind Speed in South Western Nigeria. Journal of Modern Applied Statistical Methods. 2014;13(1).

6. Mudholkar G, Srivastava D. Exponentiated Weibull family for analyzing bathtub failure rate data. IEEE Transactions on Reliability. 1993;42(2):299-302.

7. Das KK, Roy TD. On Some Length Biased Weighted Weibull Distribution. Advances in Applied Science Research. 2011;2(5): 465-475.

8. Hussain A, Ahmed PB. Misclassification in size-biased modified power series distribution and its applications. Journal of KSIAM. 2009;13(1):55-72.

9. Das KK, Roy TD. Applicability of length biased weighted generalized Rayleigh distribution. Advances in Applied Science Research. 2011;2(4):320-327.

10. Mir KA, Ahmed A, Reshi J. Structural properties of length biased beta distribution of first kind. American Jounal of Engineering Research. 2013;2(2):1-6.

11. Pandya M, Pandya S, Andharia P. Bayes estimation of Weibull lengthbiased distribution. Asian Journal of Current Engineering and Maths. 2013;2(1):44-49.

12. Mir KA, Ahmad M. Size--biased distributions and their applications. Pak J Statist. 2009;25(3):283-294.

13. Mir KA, Ahmad M. On Size-biased Geeta Distribution. Pak J Statist. 2009,11;25(3):309-316.

14. Oluyede BO. On Some Length Biased Inequalities for Reliability Measures. Journal of Inequalities and Application. 2000;5:447-466.

15. Shen Yu, Ning J, Qin J. Analyzing Length-biased Data with Semi parametric Transformation and Accelerated Failure Time Models. J Am Stat Assoc. 2009;104(487):1192-1202. 